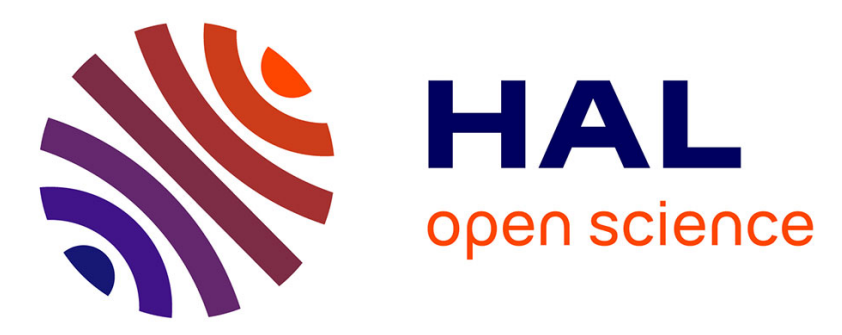

\title{
Study and prevention of cracking during weld-repair of heat-resistant cast steels
}

Traian Branza, Frederic Deschaux-Beaume, G. Sierra, Philippe Lours

\section{To cite this version:}

Traian Branza, Frederic Deschaux-Beaume, G. Sierra, Philippe Lours. Study and prevention of cracking during weld-repair of heat-resistant cast steels. Journal of Materials Processing Technology, 2009, 209, pp.536-547. 10.1016/j.jmatprotec.2008.02.033 . hal-00562399

\section{HAL Id: hal-00562399 \\ https://hal.science/hal-00562399}

Submitted on 4 Dec 2019

HAL is a multi-disciplinary open access archive for the deposit and dissemination of scientific research documents, whether they are published or not. The documents may come from teaching and research institutions in France or abroad, or from public or private research centers.
L'archive ouverte pluridisciplinaire HAL, est destinée au dépôt et à la diffusion de documents scientifiques de niveau recherche, publiés ou non, émanant des établissements d'enseignement et de recherche français ou étrangers, des laboratoires publics ou privés. 


\title{
Study and prevention of cracking during weld-repair of heat-resistant cast steels
}

\author{
T. Branza ${ }^{a}$, F. Deschaux-Beaume ${ }^{b, *}$, G. Sierra $^{b}$, P. Lours ${ }^{a}$ \\ a Université de Toulouse, Mines Albi, CROMeP, Campus Jarlard 81013 Albi Cedex 09, France \\ b Université de Montpellier 2, IUT de Nîmes, Mechanics and Civil Engineering Laboratory, 30907 Nîmes, France
}

Keywords:

Heat-resistant cast steel

Weld-repair

Cracking

\begin{abstract}
A B S T R A C T
Heat-resistant cast steels are highly sensitive to cracking as they are weld-repaired because of their very low ductility. To prevent weld-repair cracking of three different heat-resistant cast steels used for the manufacturing of superplastic forming (SPF) dies, the effect of various welding parameters, such as the choice of the filler material, the number of weld passes and the pre-heating temperature has been investigated. The choice of an appropriate filler metal and the pre-heating to $400^{\circ} \mathrm{C}$ of the material prior to welding drastically lower the propensity to cracking, but remain unable to eliminate cracks entirely. To further reduce weld-repair cracking and hopefully prevent it completely, a buttering technique has been developed. Buttering of the base metal surface with nickel alloys before weld-repair has been shown to prevent cracking of the base metal, but results in some hot-cracking of the buttering layer itself. On the other hand, buttering with Ni-Fe alloys, less sensitive to hot-cracking, results in crack-free weld-repairs.
\end{abstract}

\section{Introduction}

Due to their high-metallurgical stability, good oxidation and corrosion resistance, satisfactory creep strength, and low cost compared to nickel-base superalloys, heat-resistant cast steels are extensively used to manufacture component parts for the petrochemical industry. Since the early 1960s, steamreformer and pyrolysis tubes have been manufactured by centrifugal casting of such steels, of $25 \% \mathrm{Cr}-20 \% \mathrm{Ni}$ type (Bhaumik et al., 2002) or 25\% Cr-35\% Ni type (De Almeida et al., 2003). More recently, heat-resistant cast steels have been used for the manufacture of bulk superplastic forming (SPF) tools processed by sand-casting and machining (Montagnon et al., 2004).

Typically heat-resistant cast steels contain about $20-30 \mathrm{wt} \%$ of chromium, $20-50 \mathrm{wt} \%$ of nickel, and up to $0.45 \mathrm{wt} \%$ of carbon. The significant creep resistance of these steels is mainly related to the high-carbon content, resulting in extensive carbide precipitation. Two types of carbides form during the manufacturing process (Wu et al., 2000). Primary carbides, precipitated at the end of the solidification process in the interdendritic regions and grain boundaries, prevent intergranular sliding. A secondary precipitation, formed during the ageing treatment (stabilisation) or during service, finely distributed within the grains, reduces the dislocation mobility. The major drawback of these alloys is their very low ductility at room temperature, their elongation to rupture being as low as $2 \%$. Generally, the creep strength increases and the ductility decreases with the carbon content. In addition, the coarsening of the secondary carbides as the alloy is exposed to elevated temperature further reduces the ductility (Ray et al., 2003). For high-carbon alloys, this low ductility prevails up to temperatures in the range of $500-700^{\circ} \mathrm{C}$. This specific characteristic results in a particular behaviour of the

* Corresponding author. Tel.: +33466 6285 86; fax: +33466 628531 . 
materials regarding fracture. First, they are prone to cracking in service. This is particularly detrimental to SPF dies, subject to severe thermomechanical cycles that may initiate and propagate fatigue cracks (Branza et al., 2004b). Furthermore, the assembly by welding or the necessary weld-repair following in-service failure is very difficult and generally induces cracking, the material being unable to properly accommodate the welding thermal stresses through a proportioned plastic strain (Haro et al., 2000).

Welding is well known to induce thermal gradient and stresses, which can create distortion or cracks if the material is too brittle. The mechanism of stress generation during welding is rather complex because it depends on many factors. The thermal strain in each point of the welding component depends on the heat source induced by welding but also on the geometry and size of the component, which affect heat diffusion. The thermal gradient results in the establishment of accommodation strains and associated stresses. In the case where, locally, the yield stress is reached, some plastic strain is involved. Moreover, welding with a filler metal different from the base metal creates a heterogeneous structure likely to enhance the stress intensity factor and the resulting stress level locally. It is generally assumed that welding a material with no risk of induced crack requires a minimum ductility assessed by an elongation to rupture in tension of $4 \%$ or more (Colwell and Hoffman, 1998). In order to enhance ductility slightly if required, solution annealing to dissolve secondary carbides is recommended (Ebert, 1976). However, the annealing temperature must be very high to allow complete dissolution, i.e. above $1200^{\circ} \mathrm{C}$. Another possibility consists in using a buttering technique so as to deposit a soft intermediate layer, which can accommodate thermal strains and release stresses (Ebert, 1976), but this technique does not always prevent cracking (Haro et al., 2002).

The present paper focuses on the weld-repair processes of three austenitic heat-resistant cast steels. These materials, used for manufacturing large SPF tools (Branza et al., 2004a), are investigated in the shape of bulk parallelepipedic specimen with dimensions representative of industrial dies. A standard weld-repair technique with two different filler metals as well as the results obtained in terms of structural integrity are first presented and discussed. The effect of the type of filler material, the number of weld passes, and the pre-heating temperature, previously analysed using numerical simulation (Branza et al., 2005), are experimentally investigated in this study. Next, to further improve the feasibility of the weld-repair process, a buttering technique, using various buttering materials, is detailed and discussed. It is implemented to reduce the risk of cracking, which mainly depends on the chemical composition of the buttering material.

\section{Materials and welding processes}

\subsection{The base materials: heat-resistant cast steels}

Three austenitic heat-resistant cast stainless steels, produced by the sand-casting process, and cut into $400 \mathrm{~mm} \times 160 \mathrm{~mm} \times 120 \mathrm{~mm}$ blocks, are investigated. The size and geometry of specimens to be weld-repaired are chosen to be representative of real industrial SPF dies in terms of self-clamping effect and volume heat diffusion.

The composition (wt\%) of the three steels is given in Table 1. They are referred to as SP35, SP40 and SP50, SP standing for superplastic and designating the targeted application as SPF dies, and the associated number standing for the mean $\mathrm{Ni}$ content of the steel. The three alloys have similar $\mathrm{Cr}$ content (about $25 \mathrm{wt} \%$ ) and differ in their content in other alloying elements. SP35 contains $1 \mathrm{wt} \%$ niobium plus a small amount of other carbide-forming elements such as Mo and W. Nevertheless, the low $\mathrm{C}$ content should limit the volume fraction of carbides for this grade. SP40 contains more carbon and less $\mathrm{Nb}$, Mo and $\mathrm{W}$ than SP35, resulting in a different nature of carbides precipitated. SP50 exhibits the highest carbon content and contains about $5 \mathrm{wt} \%$ tungsten, enhancing the volume fraction of $\mathrm{W}$ carbides.

The microstructure of the alloys, revealed by scanning electron microscopy (SEM) observations (Fig. 1), consists of a typical dendritic structure, with coarse primary arms (about $200 \mu \mathrm{m}$ width), separated by carbide rich interdendritic zones. Note that both the size of the dendrite arms and the width of the interdendritric carbide network slightly differ for SP35, SP40 and SP50. The type of the primary carbides formed during solidification depends on the nature and amount of alloying elements. Energy dispersive spectrometry (EDS) analysis indicates that they are essentially chromium carbides for the three grades but that other types of carbides form, i.e. W carbides for SP50, and Nb-Mo carbides for SP35 and SP40. This confirms previous assumptions based on chemical composition considerations. Fig. $2 b-d$, is the result of an EDS analysis performed on the primary carbides in SP40 shown on the associated SEM micrograph (Fig. 2a). It reveals the presence of two types of carbides, namely chromium-rich carbides (dark contrast in the micrograph) and Nb-rich carbides (bright contrast). The centre of the dendrites also reveals the presence of a fine dispersion of secondary carbides, precipitated during the ageing treatment carried out after casting. This stabilisation treatment consists in a $24-\mathrm{h}$ exposure at $950^{\circ} \mathrm{C}$ and promotes the carbide precipitation issued from supersaturated elemental carbon remaining in excess in the solid solution.

Mechanical properties of the three alloys are presented in Table 2. They all exhibit rather low yield strength and ulti-

Table 1 - Chemical composition of the heat-resistant cast steels

\begin{tabular}{lcccccccccccc} 
& Fe & C & Cr & Ni & Mn & Si & P & S & Mo & W & Nb & V \\
\hline SP35 & bal. & 0.175 & 25.20 & 34.85 & 1.107 & 0.627 & 0.01 & 0.008 & 0.282 & 0.193 & 1.011 & - \\
SP40 & bal. & 0.313 & 24.40 & 39.2 & 1.11 & 1.45 & $<0.001$ & 0.0011 & $<0.020$ & $<0.002$ & 0.328 & 0.038 \\
SP50 & bal. & 0.458 & 26.76 & 49 & 0.98 & 1.21 & 0.008 & 0.001 & - & 4.9 & - & - \\
\hline
\end{tabular}



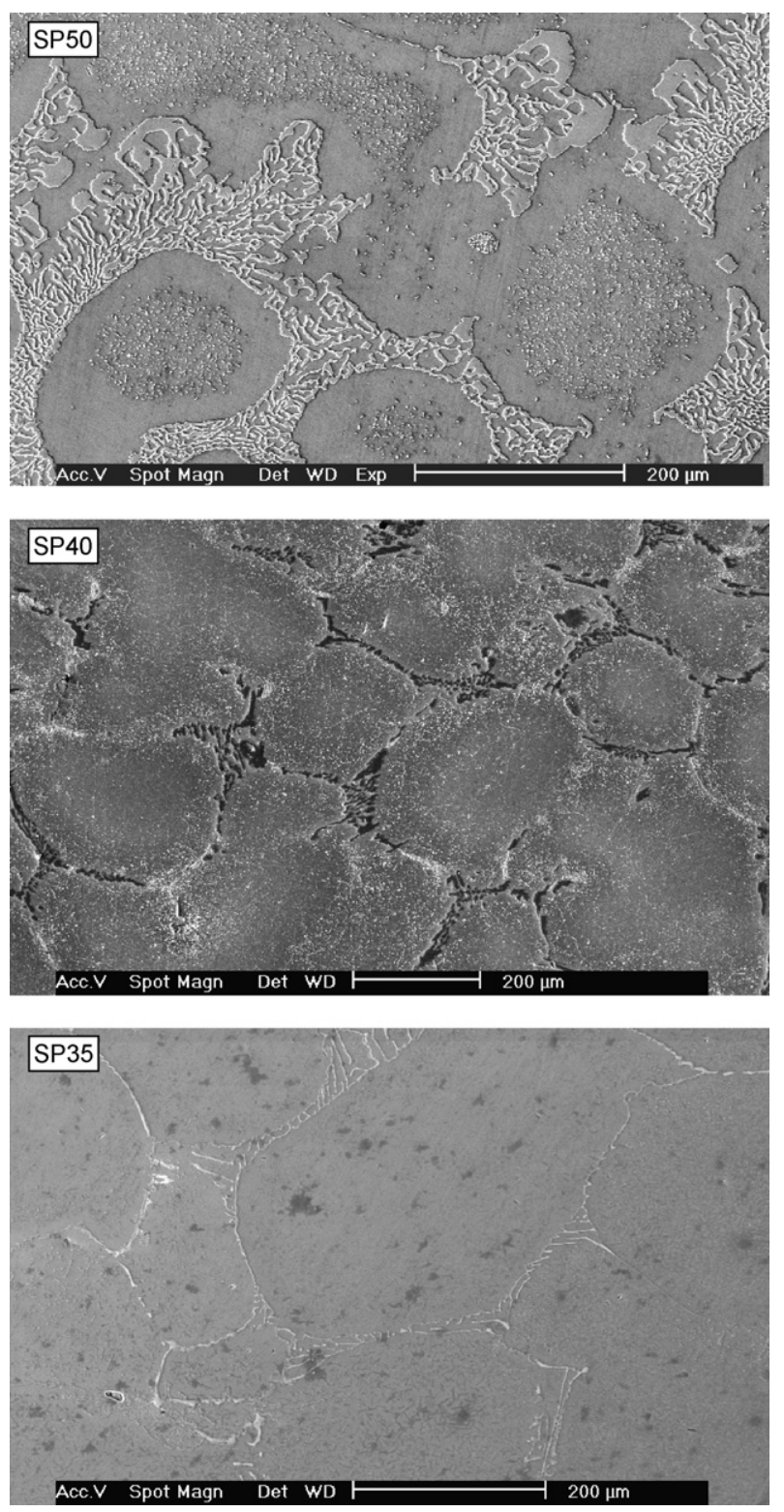

Fig. 1 - Microstructure of the heat-resistant cast steels.

mate tensile strength at room temperature and only a slight decrease of their mechanical properties as the testing temperature increases. Note that the elongation to rupture is very low for all grades, especially at room temperature. The ductility of alloys tends to increase with temperature but remains very low, in particular for SP50 grades for which elongation to rupture is similar in the whole temperature range from 20 to $750^{\circ} \mathrm{C}$.

\subsection{The filler materials}

Two types of filler materials are used:

- Heat-resistant filler alloys, with high-temperature mechanical properties similar to the heat-resistant steels, to ensure mechanical strength continuity at the interface between the welding and the base metal.

- Low-strength alloys, able to release stresses generated by welding through plastic flow. Those filler alloys are buttered using a low-energy welding process, in order to deposit an intermediate soft layer aimed at accommodating welding stress and strain before final welding with a heat-resistant filler metal (Bellrose, 1986).

Table 3 gives the chemical composition of the two heatresistant filler metals used for weld-repairs. The first one, designed by the commercial name UTP2133Mn (BölherThyssen), has a composition rather close to that of the base material and enables quasi-homogeneous welding. The second one, designated by UTP6222Mo (Bölher-Thyssen), has a chemical composition corresponding to a 625 nickel alloy and is the most standard filler material used for welding nickelbase alloys or heat-resistant austenitic steels. These two filler materials are used in the form of shielded electrodes with 250-300 mm length and $2.5 \mathrm{~mm}$ core diameter. Note that the room temperature tensile properties (yield strength and ultimate tensile strength, Table 5) of these filler materials are higher than the base metal. This has a detrimental effect on the risk of cracking of base materials.

Four buttering alloys are tested, chosen according to their mechanical properties and their chemical composition. They are austenitic nickel-rich alloys to guarantee the metallurgical compatibility with the heat-resistant cast steels to be weld-repaired. Table 4 gives the chemical composition of the four buttering alloys. The first two, Ni200 (FSH Welding) and UTP80Ni (Bölher-Thyssen) are nickel alloys with Ni content above $96 \mathrm{wt} \%$, and the last two, SAFONTE (SAF) and INVAR (Imphy Alloys) are Ni-Fe alloys. UTP80Ni and SAFONTE are used as shielded electrodes with $250 \mathrm{~mm}$ length and $2.5 \mathrm{~mm}$ core diameter, whereas Ni200 and INVAR are used in the form of $2 \mathrm{~mm}$ diameter welding wires. They are all satisfactorily ductile and have a rather low-yield stress both at room and high temperature to facilitate stress release. Table 5 gives the room temperature mechanical properties available for these

Table 2 - Mechanical properties of the heat-resistant cast steels

\begin{tabular}{|c|c|c|c|c|c|c|c|c|c|}
\hline & \multicolumn{3}{|c|}{ YS (MPa) } & \multicolumn{3}{|c|}{ UTS (MPa) } & \multicolumn{3}{|c|}{$\% \mathrm{El}$} \\
\hline & $20^{a}$ & $500^{a}$ & $750^{\mathrm{a}}$ & $20^{\mathrm{a}}$ & $500^{\mathrm{a}}$ & $750^{a}$ & $20^{\mathrm{a}}$ & $500^{a}$ & $750^{\mathrm{a}}$ \\
\hline SP35 & 232 & 154 & 124 & 308 & 246 & 239 & 2.0 & 4.0 & 7.0 \\
\hline SP40 & 220 & 175 & - & 335 & 300 & - & 2.0 & 5.0 & - \\
\hline SP50 & 259 & 198 & 170 & 373 & 304 & 299 & 2.0 & 2.5 & 2.1 \\
\hline
\end{tabular}

${ }^{\mathrm{a}} \mathrm{T}\left({ }^{\circ} \mathrm{C}\right)$. 
(a)

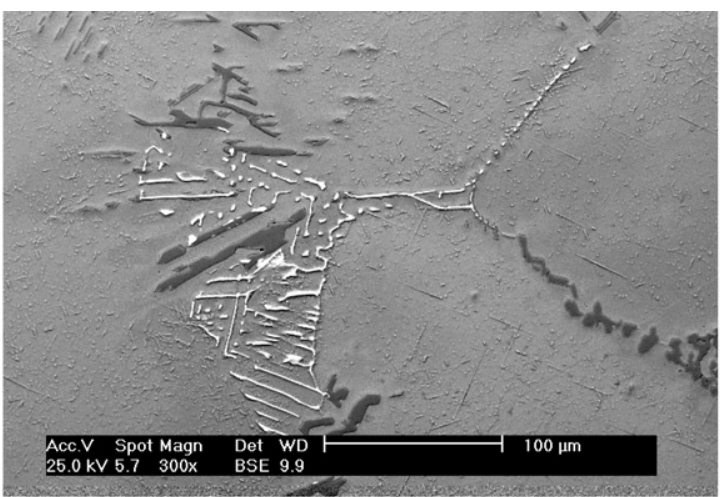

(c)

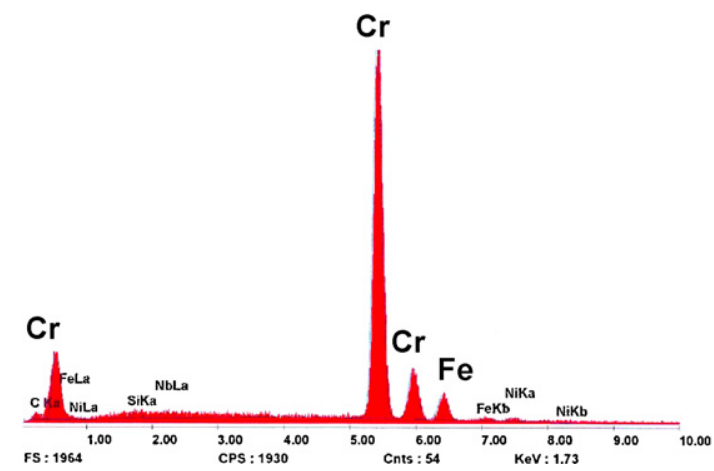

(b)

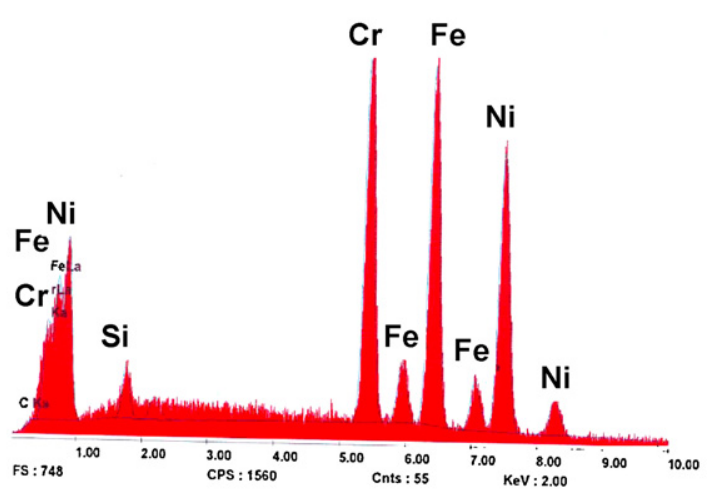

(d)

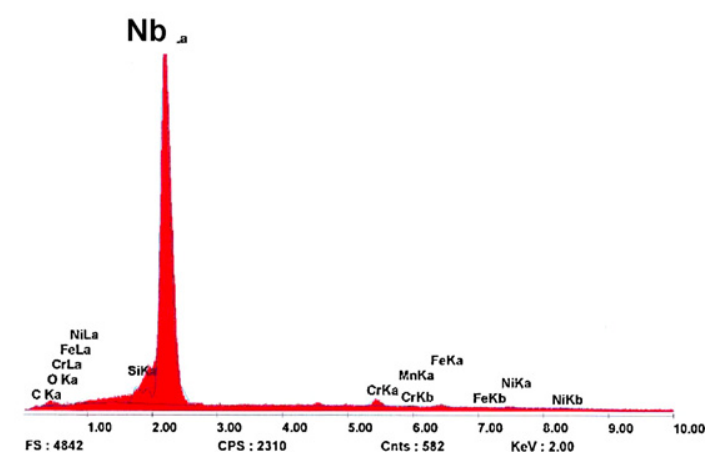

Fig. 2 - SEM micrograph of SP40 (a) and EDS analysis of the matrix (b), of the dark contrast precipitates (c) and of the bright contrast precipitates (d).

\section{Table 3 - Chemical composition of the filler metals}

\begin{tabular}{llllllllcccc} 
Filler metal & $\mathrm{C}$ & $\mathrm{Si}$ & $\mathrm{Mn}$ & $\mathrm{P}$ & $\mathrm{S}$ & $\mathrm{Ni}$ & $\mathrm{Cr}$ & $\mathrm{Mo}$ & $\mathrm{Nb}$ & $\mathrm{Fe}$ \\
\hline UTP2133Mn & $0.1-0.17$ & $\leq 0.6$ & $3.5-5$ & - & - & $32-34$ & $20-22$ & - & $1-1.5$ \\
UTP6222Mo & 0.03 & 0.3 & 0.8 & 0.01 & 0.005 & 63 & 22 & 9 & 3.5 & $<1$ \\
\hline
\end{tabular}

Table 4 - Chemical composition of the buttering metals

\begin{tabular}{lcccccccc} 
Buttering metal & $\mathrm{C}$ & $\mathrm{Ni}$ & $\mathrm{Mn}$ & $\mathrm{Si}$ & $\mathrm{Ti}$ & $\mathrm{Al}$ & $\mathrm{Fe}$ & \\
\hline UTP80Ni & $<0.02$ & solde & 0.25 & 0.8 & 2 & 0.2 & 0.1 \\
Ni200 & 0.15 & solde & 0.35 & 0.35 & & 0.4 & 0.01 \\
SAFONTE BM & & 52 & 0.35 & 0.2 & & \\
INVAR & & 36 & & & \\
\hline
\end{tabular}

four filler metals. They all have lower tensile strength than the heat-resistant filler metals. However, except for the Ni200, they have room temperature strength similar to or higher than the base materials. Although the use of buttering alloys is ben-

\begin{tabular}{|c|c|c|c|}
\hline Filler metal & YS (MPa) & UTS (MPa) & \% El. \\
\hline 2133Mn & 410 & 600 & 25 \\
\hline IN625 & 450 & 760 & 30 \\
\hline UTP80Ni & 300 & 450 & 30 \\
\hline Ni200 & 148 & & 47 \\
\hline SAFONTE & & 530-580 & \\
\hline INVAR & 260 & & \\
\hline
\end{tabular}

eficial in reducing stresses in the base material, it is not sure that it is sufficient to prevent cracking.

\subsection{The welding process}

The weld-repair tests are carried out on heat-resistant cast steel specimens of which the dimensions and shape are given in Fig. 3. Prior to welding, specimens are prepared in a similar way to the industrial dies before a weld-repair operation after in-service cracking. Indeed, a $20-\mathrm{mm}$ depth V-groove is machined on the two largest faces of the specimens, to simulate the material removal usually performed around a crack before weld-repair.

The automatic shielded metal arc welding process (SMAW) is used for operations with the heat-resistant filler metals and with UTP80Ni and SAFONTE buttering metals. During welding, 
(a)

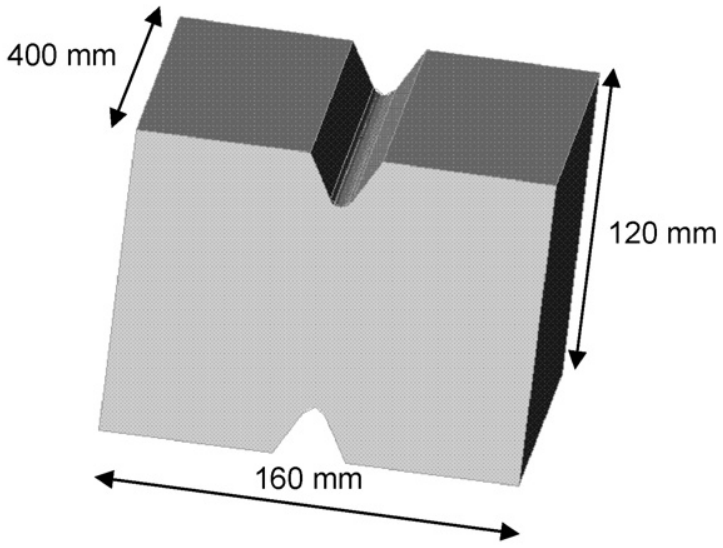

(b)

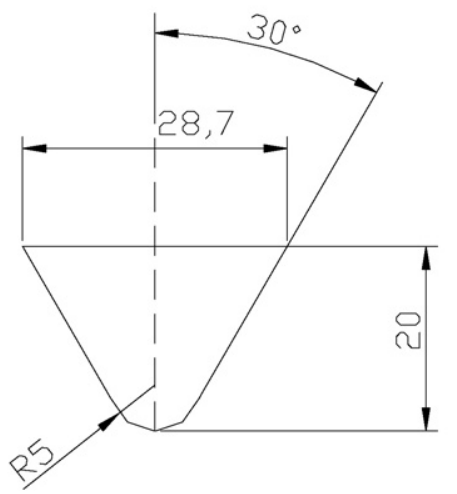

Fig. 3 - Geometry and dimensions of the weld-repair samples (a) and of the V-groove (b).

the shielded electrode is horizontally translated at a constant welding speed to fill in the V-groove, using a welding cantilever-beam. To ensure a constant arc voltage, the shielded electrode is also vertically translated while melted, using an electrically controlled powered pay-off system. Buttering with Ni200 and INVAR wires is carried out using the manual gas tungsten arc welding process (GTAW).

The welding parameters, i.e. intensity, arc voltage and welding speed, are optimised for each "base metal/filler metal" couple prior to performing weld-repair tests. Some specimens are uniformly pre-heated at $400^{\circ} \mathrm{C}$ before welding using resis-

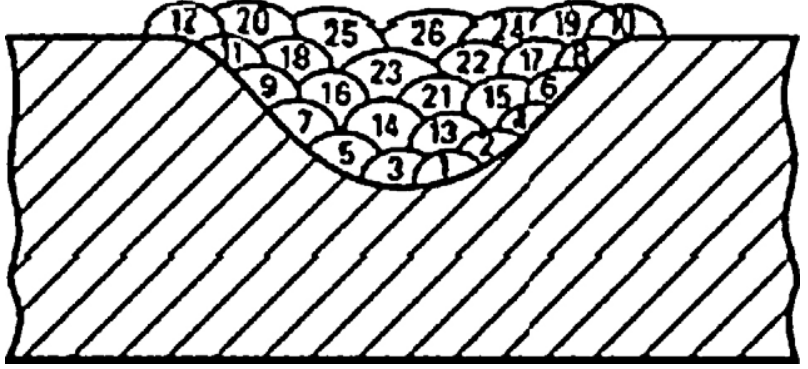

Fig. 4 - Principle of the buttering technique: passes 1-12 are buttering passes with a soft filler metal; passes 13-26 are filling passes with a heat-resistant filler metal.

tive heater. This pre-heating temperature has been chosen because it is generally considered as the maximal temperature allowing a manual weld-repair without special equipment.

Two types of weld-repair techniques are carried out:

- Standard multi-pass weld-repairs with a heat-resistant filler metal, by partially or totally filling the V-groove in several passes.

- Weld-repairs using a buttering technique (Lant et al., 2001), including initial 12 deposit passes over the V-groove surface with a low-strength buttering alloy and the subsequent complete filling with a heat-resistant filling alloy (Fig. 4).

\section{Weld-repair with standard multi-pass technique}

The standard weld-repair tests are carried-out on the three base materials, using the two filler metals, with or without pre-heating at $400^{\circ} \mathrm{C}$, and with a various number of passes, corresponding to a partial (10 and 16 passes) or complete (40 passes) filling of the V-groove. The different welding test conditions are summarised in Table 6. Subsequently to welding, cross-sections for metallographic observations are cut in the middle of each specimen to assess for possible cracking of the material.

For most welding conditions, the cross-section examinations reveal the presence of cracks, systematically located in

\section{Table 6 - Summary of welding conditions for standard multi-passes technique}

\begin{tabular}{|c|c|c|c|c|c|c|}
\hline Test no. & Base metal & Filler metal & Pre-heating temperature $\left({ }^{\circ} \mathrm{C}\right)$ & Energy Q/v (J/mm) & Passes number & Cracking \\
\hline 1 & SP35 & UTP6222Mo & 20 & 562 & 40 & Yes \\
\hline 2 & SP35 & UTP6222Mo & 400 & 562 & 10 & No \\
\hline 3 & SP50 & UTP6222Mo & 20 & 562 & 40 & Yes \\
\hline 4 & SP50 & UTP6222Mo & 400 & 562 & 10 & Yes \\
\hline 5 & SP50 & UTP6222Mo & 400 & 562 & 40 & Yes \\
\hline 6 & SP40 & UTP6222Mo & 20 & 562 & 40 & Yes \\
\hline 7 & SP40 & UTP6222Mo & 400 & 562 & 10 & No \\
\hline 8 & SP40 & UTP6222Mo & 400 & 562 & 16 & Yes \\
\hline 9 & SP40 & UTP6222Mo & 400 & 562 & 40 & Yes \\
\hline 10 & SP40 & UTP2133Mn & 20 & 585 & 40 & Yes \\
\hline 11 & SP40 & UTP2133Mn & 400 & 630 & 10 & No \\
\hline 12 & SP40 & UTP2133Mn & 400 & 630 & 16 & Yes \\
\hline 13 & SP40 & UTP2133Mn & 400 & 630 & 40 & Yes \\
\hline
\end{tabular}




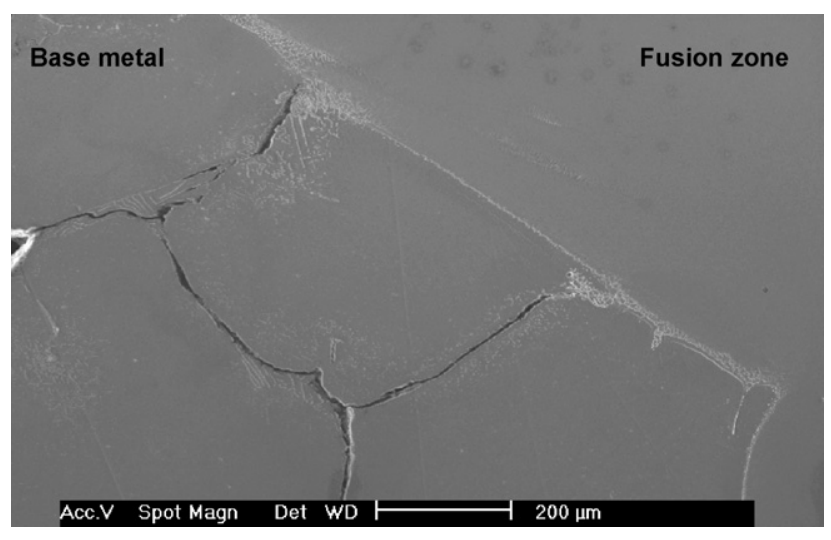

Fig. 5 - Morphology and location of cracks in the SP35 base metal after welding with UTP6222Mo filler metal and pre-heating at $400^{\circ} \mathrm{C}$.

the base metal, close to the fusion zone. However, the number and the size of the cracks depend on the base and filler materials, the number of deposited passes and the pre-heating temperature of the specimen before welding. The only experimental cases resulting in crack-free cross-sections correspond to tests performed on SP35 and SP40 grades implemented with 10 filling passes and with a pre-heating temperature of $400^{\circ} \mathrm{C}$ (Table 6). Fig. 5 shows, for SP35 pre-heated at $400^{\circ} \mathrm{C}$ and welded with UTP6222Mo, the morphology and location of the cracks. Cracks always propagate along the interdendritic primary carbides, which form a quasi-continuous network within the base metal.

In order to compare the extent of cracking after welding for the different welding conditions, the total crack length and crack surfaces on a weld-repair cross section have been measured using image analysis of low-magnification SEM micrographs $(30 \times)$ viewing the entire area adjacent to the fusion zone all over the V-groove surface. Fig. 6 presents, for various welding conditions, the total cumulative crack length within the whole affected region. Namely, the figure shows the effect of the number of passes, the nature of the base and filler metal as well as the impact of a pre-heating on the propensity of the base material to crack upon welding.

Below 10 weld-repair passes deposited with UTP6222Mo over pre-heated SP40, no crack propagates. As indicated in Table 6, the same result is obtained for pre-heated SP40 and SP35, respectively weld-repaired with UTP2133Mn and UTP6222Mo. Pre-heated SP40 welded with UTP6222Mo becomes sensitive to cracking between the tenth and the sixteenth pass as shown in Fig. 6a. Beyond 16 passes, cracks further propagate or new cracks initiate resulting in an increased total crack length reaching its maximum value as the entire filling of the V-groove is completed (40 passes). These preliminary experimental observations confirm the results related in a previous work (Branza et al., 2004a) showing an increase of the calculated maximal stress as welding passes cumulate in the frame of a multi-pass process.

Fig. $6 \mathrm{~b}$ shows the influence of the base material on the propensity to cracking. Amongst the three base materials, SP50 exhibits the largest extent of cracks in perfect concordance with its high brittleness maintained up to high temperature (Table 2). SP50 is also the only base material to be cracked after 10 passes (Table 6). Welding SP35 and SP40 results in similar crack damages as a consequence of a similar ductility and thermomechanical behaviour. Note however that the slightly lower crack extent in SP40 is probably due to its slightly higher ductility at high temperature. (a)

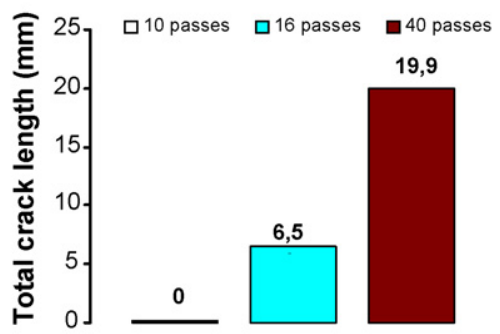

(c)

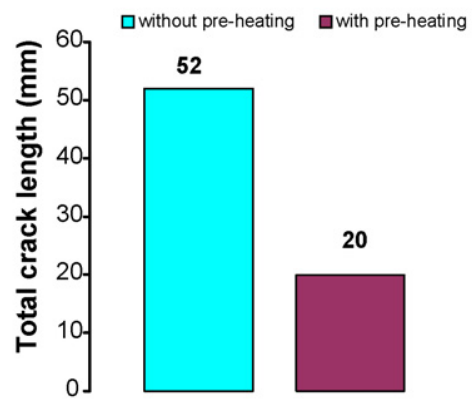

(b)

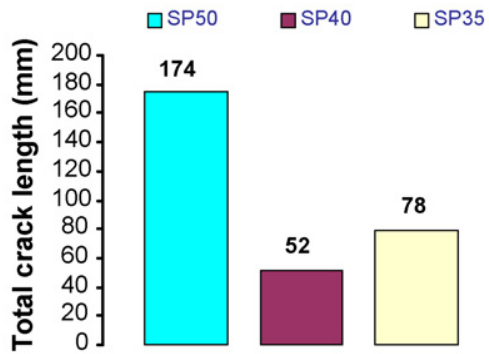

(d)

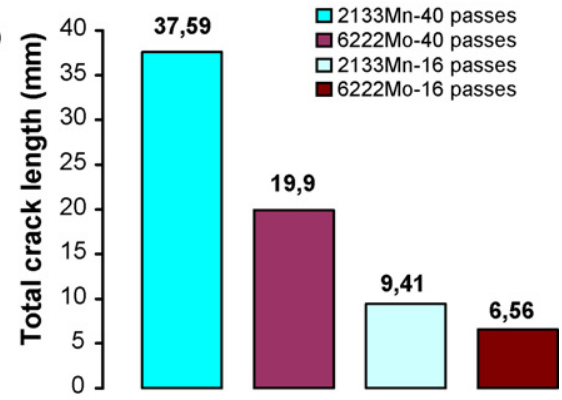

Fig. 6 - Influence of welding conditions on the total crack length: (a) effect of the number of passes (welding of SP40 with UTP6222Mo filler metal and pre-heating); (b) effect of the base metal (40 passes welding with UTP6222Mo filler metal without pre-heating); (c) effect of the pre-heating (40 passes welding of SP40 with UTP6222Mo filler metal); (d) effect of the filler metal (welding of SP40 with pre-heating). 
Fig. 6c shows the effect of pre-heating SP40 on crack development measured after the complete filling of the groove with UPT6222Mo filler metal. Though pre-heating at $400^{\circ} \mathrm{C}$ cannot prevent cracking completely, it decreases the total length of the cracks that develop within the base material significantly. Consequently, a higher pre-heating temperature can be assumed to be appropriate in further decreasing the risk of damage and possibly preventing the base material from cracking. However, such a solution is not industrially viable as this would render die handling very difficult and would increase the overall cost of weld-repair. As indicated in Fig. 6d, the choice of the filler material is also of utmost concern. Depending on the nature of the filler metal, the total crack length for pre-heated SP40 repaired either with 40 passes or 16 passes may vary significantly. In both cases, the use of UTP6222Mo results in lower crack length than the use of UTP2133Mn, suggesting that higher stress develops in the base material in the latter case. However, the higher energy required to obtain a good melting of the UTP2133Mn electrode should decrease the thermal transient, and then be beneficial in reducing thermal stresses.

\section{Weld-repair using preliminary buttering}

The choice of an adapted filler metal, associated to an appropriate pre-heating of the base material, has clearly been demonstrated to be able to reduce the formation of weldinduced cracks drastically. However, these two actions alone are not sufficient to fully prevent cracking of the weld-repaired base heat-resistant cast steels. This is true for the three grades investigated, though their propensity for cracking may be slightly different depending on their intrinsic medium and high-temperature ductility. In order to further improve the behaviour of materials regarding their ability to limit and avoid the generation of weld-induced cracking, an initial surface buttering is carried out prior to the final filling of the V-groove. The buttering technique is implemented in the case of the SP40 grade pre-heated at $400^{\circ} \mathrm{C}$. It consists in depositing a 2-3 $\mathrm{mm}$ fine layer of a dedicated alloy over the entire surface of the groove. Subsequently, weld-repair is completed by filling the groove using UTP6222Mo filler metal. Two types of buttering alloys, giving rather different results, are tested, namely nickel alloys (Ni200 and UTP80Ni) and nickel-iron alloys (SAFONTE and INVAR), the composition of which is given in Table 4.

\subsection{Buttering with nickel alloys}

When using Ni200 and UTP80Ni buttering alloys, no crack is observed in the base metal following the complete filling of the groove. This suggests that the buttering layer, despite its room temperature higher yield strength compared to the base materials in the case of UTP80Ni, is efficient for accommodating the thermal strain and release stress generated by the welding process. This could be due to the fact that during the buttering, or the subsequent welding, the buttering layer is always at a higher temperature that the base metal, then its yield strength is lower.
However, as indicated in Fig. 7 showing the cross-section of a processed specimen, cracks develop within the buttering layer. The high ductility at room temperature of these buttering alloys (Table 5) rule out the possibility of a cold cracking phenomenon. These cracks, seemingly located along the interdentritic regions of the buttering layer, may form either during solidification or later as the solidified buttered layer cools down because of the thermal stress and the segregation of elements forming detrimental brittle phases. Nickel alloys are well known for being highly sensitive to hot-cracking during welding, even though embrittlement mechanisms are not fully understood. The morphology of the cracks is nevertheless quite different depending on the chemical composition of the buttering alloy. For UTP80Ni, cracks remain fine and rather rare (Fig. 7a), whereas for Ni200, which is quasi-pure $\mathrm{Ni}$, cracks are numerous and large (Fig. $7 \mathrm{~b})$. The significant width of cracks formed in the Ni200 buttering layer, lying more or less parallel to the interfaces, indicates that the initial cracks are enlarged as a consequence of the deposition of the filling passes following the buttering.

\subsection{Buttering with nickel-iron alloys}

Alloys containing less nickel (respectively 52 and $36 \mathrm{wt} \%$ for SAFONTE and INVAR) and correlatively much more iron (respectively 48 and $63 \mathrm{wt} \%$ for SAFONTE and INVAR) are also attractive candidates for buttering heat-resistant cast steels. Lowering the nickel content should limit the risk of hot-cracking of the buttering scale. This is experimentally con-

(a)

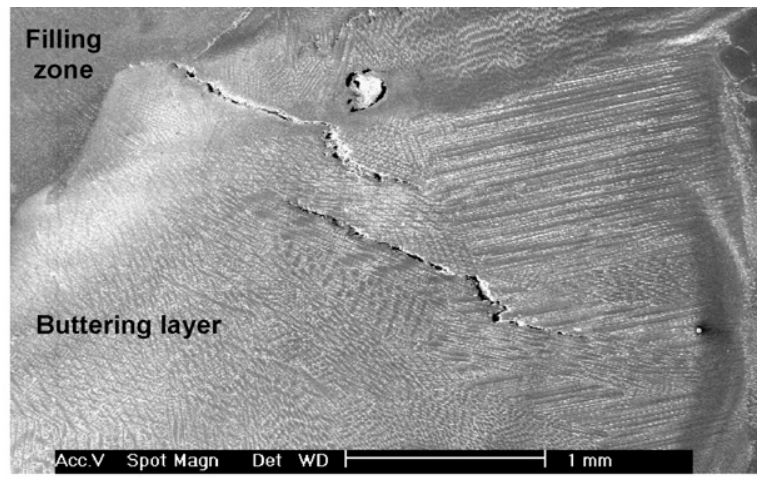

(b

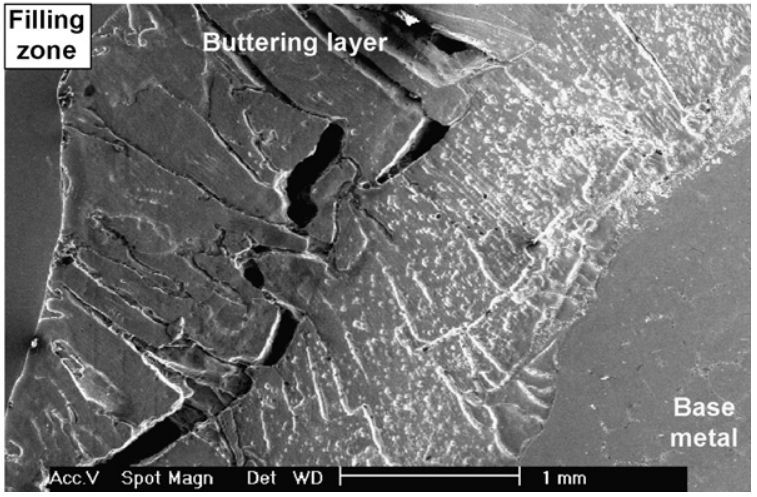

Fig. 7 - Cracking of the buttering layer following buttering with Ni alloys (SEM micrographs): (a) with UTPNi80; (b) with Ni200. 


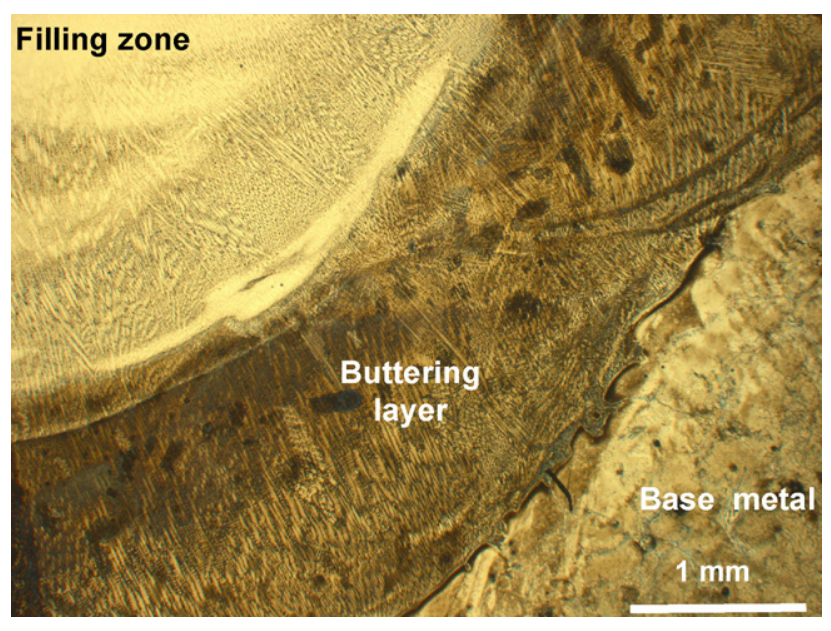

Fig. 8 - Optical micrograph of a weld-repair obtained with SAFONTE buttering alloy.

firmed by the metallographic analysis related in Fig. 8 showing the microstructural integrity of the SAFONTE buttering layer where no crack is observed. Note that, similarly to the case of buttering with quasi-pure nickel discussed in the previous section, the base material is also free of cracks after the filling of the groove is completed.

Consequently, it can be concluded that the weld-repair of brittle heat-resistant cast steels is feasible provided the process is conducted with some precautions. This includes a pre-heating of the base material, typically to $400^{\circ} \mathrm{C}$, and the interposition of a buttered layer between the base material and the filler metal used for the weld-repair final operation. This buttering layer, as well as the filling passes, must be deposited using appropriate materials. The ductile buttering layer, acting as a mechanical interface able to sustain stress and strain generated by the welding process without suffering low-temperature cracking, should be constituted of nickel-iron alloys with nickel content lower than, say 52\%, to avoid prejudicial hot-cracking.

\section{Discussion}

The weld-repair tests reveal the great sensitivity to cracking of the heat-resistant cast steels investigated. The mechanism involved is a typical solid-state cracking, due to the low ductility of the base materials and to the high-thermal stress reached during welding. The previous sections have shown unambiguously the important and critical effect of the process operating parameters on the extent of the crack that develops within the base material after welding. This section is dedicated to the analysis of the mechanisms of crack formation in the heat-resistant cast steels based on considerations of the influence of the specific chemical compositions and microstructure of the materials and the effect of the major welding parameters. In particular, the impact of the nature of the filler metals, the operation of buttering and the preheating temperature on the feasibility of weld-repair will be examined.

\subsection{General failure mechanism in the base metal}

Because of the low ductility of the heat-resistant cast steels, retained up to high temperature, namely $500-750^{\circ} \mathrm{C}$, it is assumed that the failure mechanisms resulting from the initiation and propagation of cracks are similar in the whole range of temperature. The low ductility of the base materials is mainly due to the presence of large carbides arranged in the form of a more or less continuous network within the interdendritic regions and grain boundaries. This network, directly issued from the solidification of the cast materials, may be viewed as an extremely hard and brittle skeleton (e.g. $\mathrm{HV}=800$ in the case of SP50) immersed in a rather ductile austenitic matrix (e.g. HV=260 in the case of SP50), giving rise to a composite microstructure mechanically heterogeneous. The carbide network, when subjected to stress generated by the thermal transient due to welding, is unable to properly deform plastically and suffers fracture that can be slightly limited by pre-heating the material prior to processing.

A convenient means of simulating experimentally the conditions of crack formation during welding and analysing the microstructural features responsible for crack initiation and propagation consists in loading a specimen under the electron beam of the scanning electron microscope. This so-called in-situ tensile test is performed for the SP50 grade under uniaxial tension, with samples of $2 \mathrm{~mm} \times 2 \mathrm{~mm}$ cross section and $20 \mathrm{~mm}$ length. Fig. 9 presents the related sequence of deformation. The initial microstructure of the unstrained material, free of any defect, is shown in Fig. 9a. The microstructure in Fig. 9b, corresponding to a tensile load of $1240 \mathrm{~N}$ (310 MPa), shows the occurrence of cracks precisely located along the carbide network. Prior to cracking, no uniformly distributed plastic deformation is noticeable within the investigated zone in relation to the low ductility of the material. In fact, the first cracks appear for a tensile stress of $273 \mathrm{MPa}$, just above the yield strength measured from full-size tensile tests (259 MPa, Table 2). A further increase of the load up to $1300 \mathrm{~N}$ (325 MPa) results in the complete failure of the specimen (Fig. 9c) as the degradation locally concentrates in the zone where the longest crack has developed or in a zone where several medium length neighbouring cracks coalesce. Note that the failure tensile strength measured from in-situ test is rather low compared to the ultimate tensile strength measured from full-size tensile tests $(373 \mathrm{MPa})$. This could be due to the small section of in-situ tensile samples, compared to the grain size, which induces some scattering in tensile properties. In the final stage of deformation, a slight plastic deformation is visible in the immediate vicinity of the fracture surface. This probably results from the sudden increase in stress, as the final fracture establishes, which deforms locally the ductile austenitic matrix. The in-situ experiment performed in high carbon, high nickel SP50 shows that the propagation of cracks is entirely confined within the low-toughness carbide network that fully controls the mechanical and fracture behaviour of the material. Indeed in this material, the high carbon content $(0.458 \mathrm{wt} \%)$ results in a high-volume fraction of primary carbides. As a consequence, the primary carbide network is quasi-continuous and stands for an easy path for crack propagation. In addition, the large volume fraction of secondary intragranular carbides also contributes to the high brittleness 
(a)

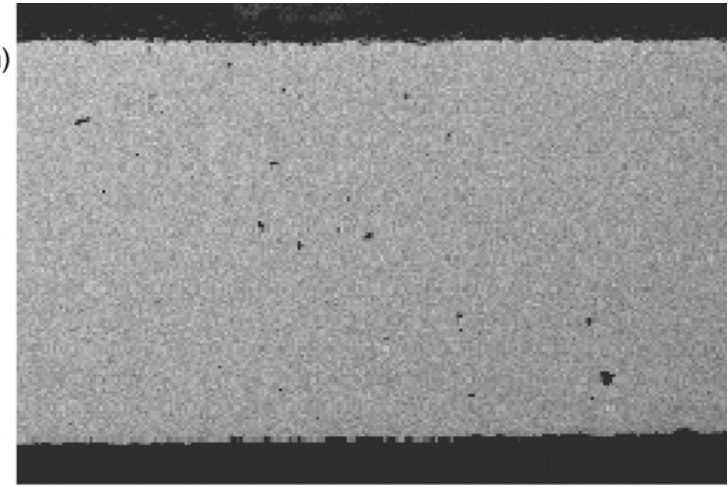

(b)

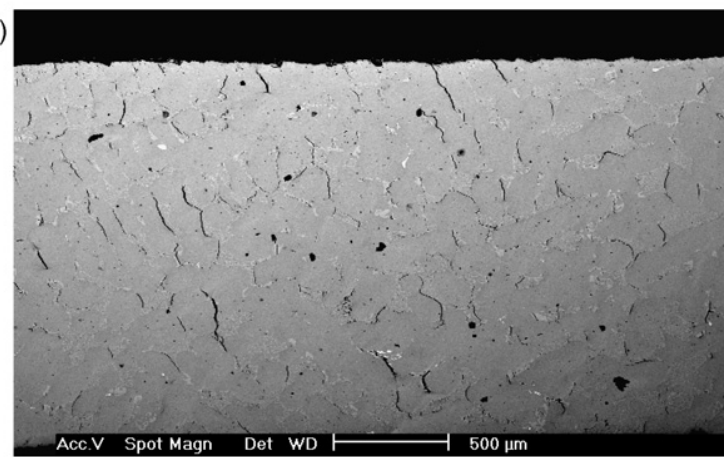

(c)

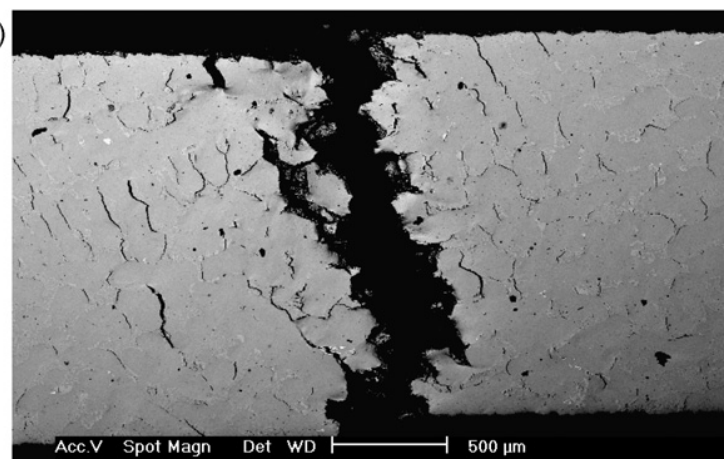

Fig. 9 - Multi-cracking phenomenon into the primary carbide network and failure of the SP50 grade (SEM in-situ tensile test): (a) unstrained material; (b) after a tensile load of $124 \mathrm{daN}$; (c) after a tensile load of $130 \mathrm{daN}$.

of the material by blocking the dislocation motion, thus limiting the plastic flow.

In the case of SP35 and SP40, the carbon content is lower, respectively 0.175 and $0.313 \mathrm{wt} \%$, leading to a lower volume fraction of primary carbides than for SP50. This can satisfactorily explain the behaviour difference between SP35/SP40 and SP50 regarding their ability to be weld-repaired, as discussed previously. However, the volume fraction of primary carbides is not the only pertinent parameter to take into account to analyse the fracture mechanisms. Indeed, though SP40 contains more carbon than SP35, its $500^{\circ} \mathrm{C}$ ductility is slightly better. This can be explained by the nature and the morphology of the carbide network. In SP35, primary carbides are particularly fine and elongated. They form a quasi-continuous network, similar from this point of view, to that prevailing in SP50. Conversely, in SP40, they show (a)

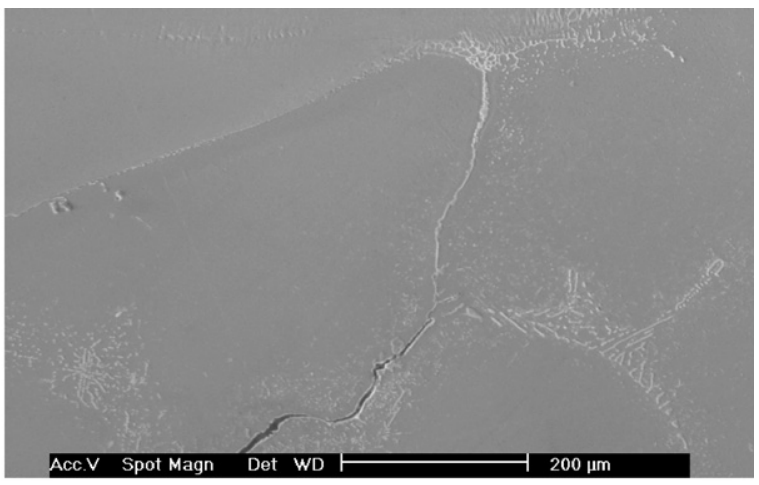

(b)

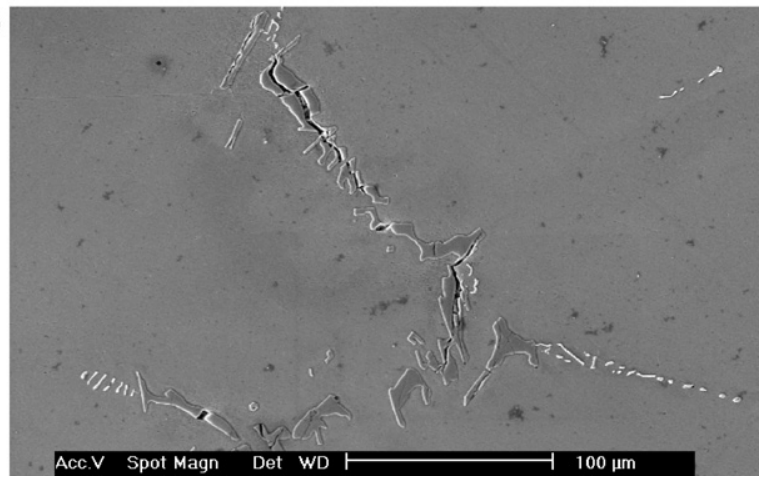

Fig. 10 - Aspect of crack in the SP35 grade (a) and SP40 grade (b).

a lower aspect ratio and precipitate in the form of coarse particles partially disconnected from each other (Fig. 1). In addition, the chemical nature of carbides is different in connection with the composition of alloys. In SP40, containing low niobium, compact primary chromium carbides preferentially form upon solidification. For niobium-rich SP35 (1 wt\%), primary carbides are either chromium or niobium with highaspect ratio showing elongated needle-shape morphology. In relation with the specific chemistry and morphology of those carbides, the morphology of cracks changes from a continuous aspect along elongated niobium carbides in SP35 (Fig. 10a), to a discontinuous aspect across isolated $\mathrm{Cr}$ carbides in SP40 (Fig. 10b).

\subsection{Cracking mechanism in the base metal upon standard welding}

Due to significant difference in their overall chemical composition, namely in carbon content and nature and content of carbide-forming elements, SP35, SP40 and SP50 show different sensitivity to weld-induced cracking. Among the three alloys and according to Fig. 6, SP50 and SP40 exhibit respectively the highest and the lowest propensity to cracking upon welding.

Primarily, cracks initiate as a consequence of the large thermal stress established in the base material during welding and the associated inability of the material to plastically deform. However, another factor, connected to the difference in microstructural and mechanical properties between the base material and the filler alloys, can play an important role in the generation of damaging stress. The stress concentra- 


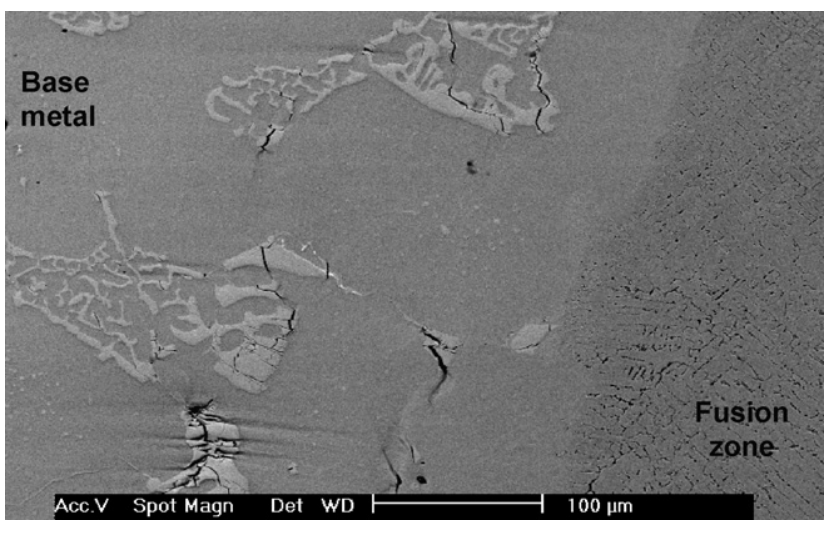

Fig. 11 - SEM in-situ tensile test on welded sample (SP50 with UTP6222Mo).

tion resulting from such a discontinuity is prone to add and superpose to the unavoidable thermal stress, thus increasing the extent of cracking. In-situ tensile tests were performed on a welded specimen to address the mechanism of failure and compare to those prevailing in the base material. In such a case, tensile loading is conducted on a bi-material including the juxtaposition of a brittle alloy with a coarse microstructure (SP50) and a more ductile material with a fine microstructure promoted by the high-cooling rate of the fusion zone (UTP6222Mo). Fig. 11 shows the characteristic of the specimen failure subsequent to tensile straining as the threshold stress leading to carbide fracture is reached. The tensile stress provokes appreciable damage only in the base metal shown on the left-hand side of the SEM micrograph. The failure is perfectly similar to that encountered in unwelded specimens, i.e. affecting preferentially the eutectic primary carbides with no plastic deformation of the austenitic matrix. The fusion zone, characterised by a very fine dendritic microstructure and shown on the right-hand side of the micrograph, remains fully uncracked. Note that the rupture of the carbide network is very close, about $100 \mu \mathrm{m}$, to the interface between the fusion zone and the base material. Away from the interface, within the base material, the specimen is free of cracks indicating the major role of the interfacial zone and its specific properties in the increase of the stress intensity.

In turn, the mechanical characteristics of the filler material can greatly affect the sensitivity to cracking of the base metal, by favouring high stress if its yield strength is high, or favouring stress concentrations if the difference in the mechanical strength between the base and filler metals is important. The use of UTP2133Mn filler material results in a larger extent of cracking than the use of UTP6222Mo. Nevertheless, the room temperature yield strength of UTP2133Mn $(410 \mathrm{MPa})$ is lower than that of UTP6222Mo $(450 \mathrm{MPa})$ (BölherThyssen, 2004). This is confirmed by Vickers micro-hardness measurements in cross-sections of weld-repaired SP40 samples, indicating a hardness of 220 and $250 \mathrm{Hv}$ in UTP2133Mn and UTP6222Mo fusion zones, respectively and $180 \mathrm{Hv}$ in the base material. In addition, the energy required to ensure a correct melting of the UTP2133Mn electrode is higher that the energy required for UTP6222Mo, which could decrease the severity of the thermal transient.
However, cracks probably form before the specimen reaches room temperature in a high to medium temperature range, and considering the high C content of UTP2133Mn, this material should have a higher yield strength than UTP6222Mo.

The last parameter affecting the sensitivity to cracking during standard welding is the pre-heating. The beneficial effect of pre-heating, well known to avoid weld-cracking in low-ductile materials, has already been shown (Branza et al., 2005), and the present results confirm the major impact of this parameter. Mainly, pre-heating reduces the thermal gradient and the associated thermal stress generated by welding and favours stress release through plastic deformation owing to the lower yield strength and higher ductility of materials at high temperature.

\subsection{Cracking mechanism in the buttering layers}

As indicated above, the interposition of a soft ductile buttering layer weld deposited between the base material and the filling metal may be highly beneficial to avoid cracking. However, the buttered layers may suffer hot cracking, phenomenon commonly observed when welding nickel-base alloys or austenitic steels. The occurrence of cracks depends on various process parameters, as well as on the chemical composition of the materials involved. The critical effect of both sulphur and phosphorus, forming low-melting point compounds, is well known (Matsuda et al., 1982). It is generally considered that in nickel-based alloys presenting a very low S solubility, the sulphur plus phosphorus content must be lower than $0.01 \mathrm{wt} \%$ to prevent hot cracking (Suutala et al., 1979). These elements segregate at the interdendritic zones, either forming low-melting point or brittle phases. The addition of Mn limits the prejudicial influence of S (Li and Messler, 2002). Other elements such as $\mathrm{Al}$, $\mathrm{Ti}$, or $\mathrm{Nb}$ may also have a detrimental effect on the hotcracking sensitivity of austenitic stainless steels (Shankar et al., 2000).

As previously discussed, the propensity to cracking and the final overall extent of cracks is much more pronounced in buttered Ni200 than UTP80Ni. Cross-sections of the two buttered alloys were observed following deposition and prior to the final welding with the filler metal UTP6222Mo. When using Ni200, cracks form straightforwardly as the alloy is buttered. On the contrary, cracks do not form when buttering UTP80Ni. Their initiation and growth require the subsequent stacking of the filling passes with UTP6222Mo.

Regarding the nature of the alloying elements of the two buttering alloys, which may dramatically impact cracking behaviour, the main difference originates in the titanium content (2 wt\% in UTP80Ni against about zero in Ni200) and to a lower extent in the aluminium content.

The EDS analysis of the interdendritic regions of UTP80Ni buttering layer shown in Fig. 12, clearly indicates the presence of Ti- and $\mathrm{Nb}$-rich phases. Undoubtedly, titanium comes from the buttering layer itself, whereas niobium must have diffused from the melted solid solution or the eutectic carbides of the base metal. Because of their intrinsic brittleness, those phases stand for preferential sites to initiate cracking. An illustration of the direct relationship between the presence of a Ti-rich phase and the occurrence of a crack is given in Fig. 13, showing a micrograph of cracks and the associated EDS 


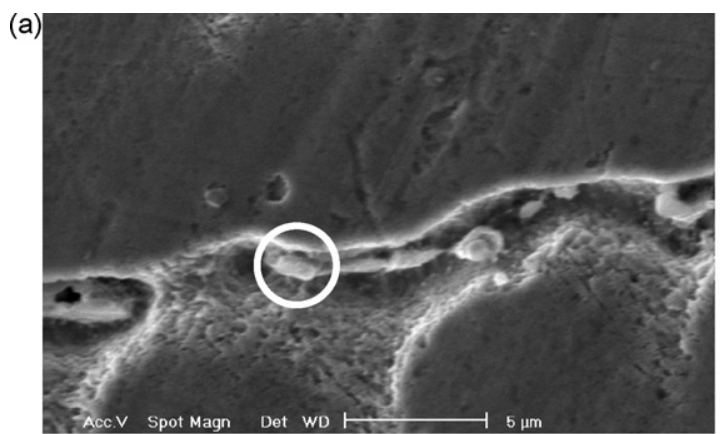

(b)

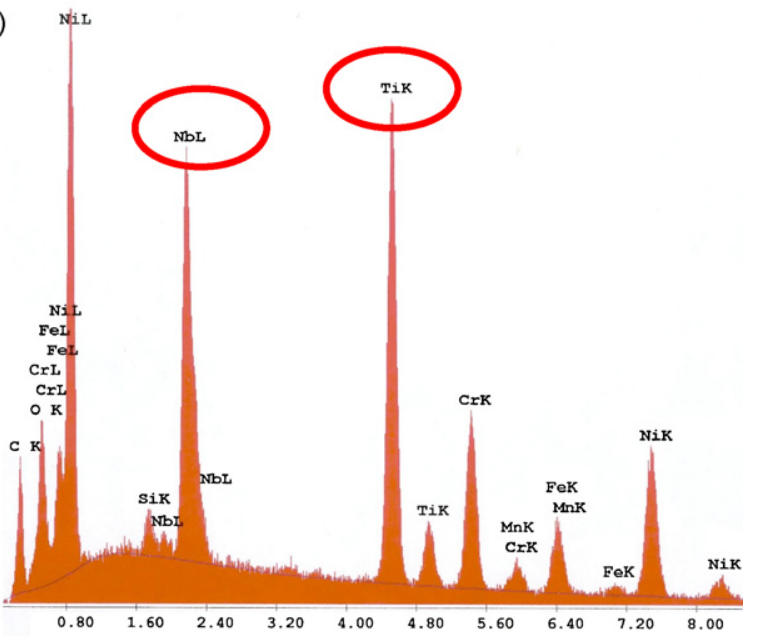

Fig. 12 - SEM micrograph (a) and EDS analysis (b) of interdendritic precipitates in the UTP80Ni buttering layer.

$\mathrm{X}$-ray image of Ti. The figure reveals a strong Ti segregation along the primary crack path and a slighter, though significant, segregation on secondary cracks. Shankar et al. (2000) have already observed the detrimental effect of Ti, explained by the formation of Ti carbides or carbo-sulphide phases in the interdendritic regions.

On the other hand, even though cracking occurs in Ni200 buttering layer, no segregation is observed. However it is known that the system Ni-NiS shows a low-melting eutectic point around $630^{\circ} \mathrm{C}$ (Shankar et al., 2003), which can promote hot cracking. It is also possible that sulphur segregates in the interdendritic regions and forms a low-melting point thick film that favours cracking. Unfortunately, this assumption cannot be confirmed as the S content in the alloy is too low to be properly analysed by EDS.

According to this assumption, the absence of cracks in the UTP80Ni buttering layer prior to final welding should be due to a beneficial effect of titanium in $\mathrm{Ni}$, which retains sulphur by forming phases with higher melting point than the Ni-NiS eutectic compound. However, these phases, formed while buttering, are highly brittle and can provoke the initiation of cracks, later during the weld-repair process, under the effect of the thermal stresses involved for instance during the filling with UTP6222Mo. Complementary analysis is however necessary to confirm these assumptions. Indeed, it is well known that only a very little change in sulphur content can greatly modify the sensitivity to hot-cracking which can also explain (a)

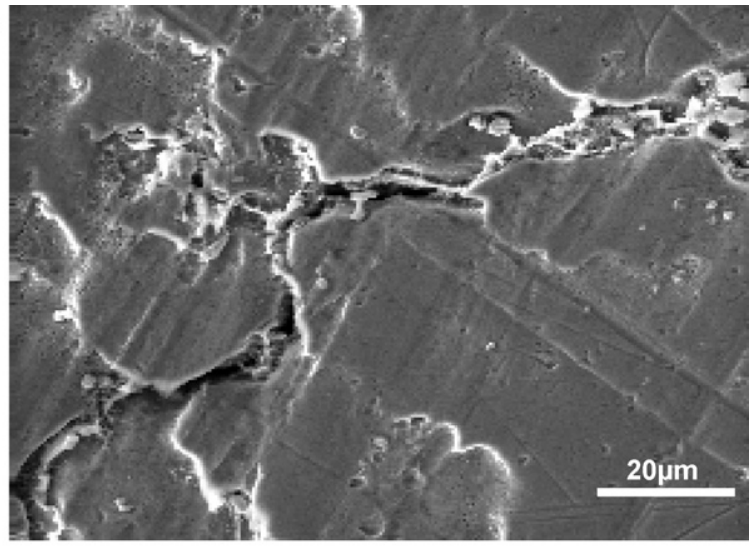

(b)

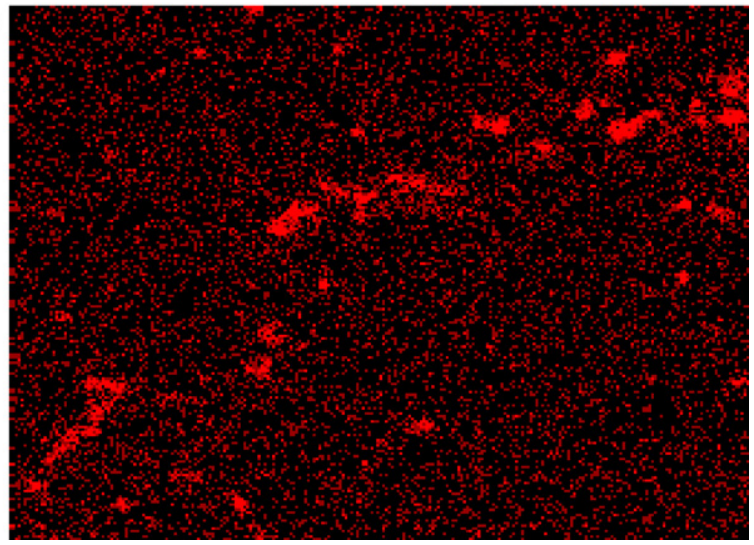

Fig. 13 - SEM micrograph (a) and EDS X-ray map (b) showing Ti segregation along the crack path in the UTP80Ni buttering layer.

the difference observed between the two nickel-base buttering alloys.

\section{Conclusion}

Among other applications, heat-resistant cast steels are employed for manufacturing superplastic forming dies and suffer severe thermomechanical cycles leading to possible stress-induced failure. This requires frequent repair of the material in order to extend the service life of the dies. This paper presents investigations on the feasibility of weld-repairing three heat-resistant cast steels with different chemical compositions, using the SMAW process. The major problem for conducting a proper defect-free weld-repair comes from the very low ductility of those alloys in the whole range of temperature involved in welding. Under the effect of the stress originated in the thermal gradient issued from welding, cracks initiate and propagate in the material along the quasi-continuous network of primary carbides characteristic of the alloy microstructure. Though present in all three materials, these cracks show various extents depending on the chemical composition of the alloy and the precise nature and morphology of the eutectic carbides. In order to prevent the crack formation, several ways are investigated.

First, a standard multi-pass welding technique is implemented and the influence of varying the welding conditions 
on the propensity to cracking is analysed. The following conclusions can be drawn:

- The crack sensitivity greatly depends on the volume fraction and the nature of the primary carbides that form upon solidification of the heat-resistant cast steels. These two parameters are both in direct correlation with the $\mathrm{C}$ content and the type and content of alloying elements.

- The mechanical properties of the filler material at room temperature, but above all at high temperature, have a strong effect on the generation of welding cracks. To better accommodate weld-induced stress, rather ductile alloys with a yield strength lower than that of the base metal are recommended.

- Cracking occurs only after a minimum number of passes has been completed. This suggests that small extent repair required by slight damage of the material can be performed with no risk of cracking.

- Pre-heating the base material up to $400^{\circ} \mathrm{C}$ drastically limits the extent of cracking by strongly reducing the magnitude of the thermal gradient established within the material. However, pre-heating alone is insufficient to fully prevent cracking.

Standard welding technique, even optimised using appropriate materials and process parameters, fails to properly repair high-nickel, high-chromium low-ductile heat-resistant cast steels. As a consequence, a buttering technique, coupled to a $400^{\circ} \mathrm{C}$ pre-heating of the base metal, has been developed. The use of a nickel-rich buttering alloy prevents cracking of the base metal occurring at medium temperature when the material cools down subsequent to welding. However, in such a case, hot cracking develops within the buttering layer itself. This phenomenon is related to the content in alloying elements of the buttering alloy, namely titanium and aluminium. It is shown that lowering these two elements, as well as controlling the amount of trace elements such as sulphur, is prone to reduce hot cracking. On the other hand, buttering with a $\mathrm{Ni}-\mathrm{Fe}$ alloy, much less sensitive to hot-cracking permits to obtain weld-repair entirely free of cracks, showing the feasibility of weld-repairing heat-resistant cast steels. This requires circumventing the difficulty brought up by the extremely low ductility of the materials by (i) choosing appropriate filler metals, (ii) pre-heating prior to welding and (iii) interposing a buttered layer deposited with mechanically and chemically suitable alloys.

\section{REFERENCES}

Bellrose, A., 1986. The efficacy of buttering in the prevention of lamellar tearing. Austral. Weld. J. 31, 3.

Bhaumik, S.K., Rangaraju, R., Parameswara, M.A., Bhaskaran, T.A., Venkataswamy, M.A., Raghuram, A.C., Krishnan, R.V., 2002. Failure of reformer tube of an ammonia plant. Eng. Fail. Anal. 9, 553-561.
Bölher-Thyssen, 2004. Technical Handbook of Welding Products.

Branza, T., Duchosal, A., Fras, G., Deschaux-Beaume, F., Lours, P., 2004a. Experimental and numerical investigation of the weld repair of superplastic forming dies. J. Mater. Process. Technol. 155/156, 1673-1680.

Branza, T., Martinier, A., Duchosal, A., Deschaux-Beaume, F., Bernhart, G., Lours, P., 2004b. Fatigue damage and weld repair of heat-resistant cast steel SPF dies. In: Proceedings of the 3rd European Conference on Super Plastic Forming, Albi, France, pp. 133-138 (Cépadues-Editions).

Branza, T., Duchosal, A., Bordreuil, C., Deschaux-Beaume, F., Fras, G., Lours, P., 2005. Influence of the welding processing on weld repair quality of SPF tools. In: Proceedings of the 4th European Conference on Superplastic Forming, Manchester, UK, pp. 121-126 (IOM Communications).

Colwell, R.L., Hoffman, J.J., 1998. Weld cracking in modified heat-resistant castings, a microstructural investigation. In: Proceedings of the NACE International Annual Conference Corrosion 98, Paper 423. NACE International.

De Almeida, L.H., Ribeiro, A.F., Le May, I., 2003. Microstructural characterization of modified $25 \mathrm{Cr}-35 \mathrm{Ni}$ centrifugally cast steel furnaces tubes. Mater. Charact. 49, 219-229.

Ebert, H.W., 1976. Fabrication of HK-40 in the field. Weld. J., 939945.

Haro, S., Lopez, D., Velasco, A., Viramontes, R., 2000. Microstructural factors that determine the weldability of a high Cr-high Si HK 40 alloy. Mater. Chem. Phys. 66, 90-96.

Haro, S., Colas, R., Velasco, A., Lopez, D., 2002. Study of weldability of a Cr-Si modify heat-resistant alloy. Mater. Chem. Phys. 776, 831-835.

Lant, T., Robinson, D.L., Spafford, B., Storesund, J., 2001. Review of weld repair procedures for low alloy steels designed to minimize the risk of future cracking. Int. J. Press. Vessels Piping 78, 813-818.

Li, L., Messler, R.W., 2002. Segregation of phosphorus and sulphur in heat-affected zone hot cracking of type 308 stainless steel. Weld. J. 81, 78-84.

Matsuda, F., Nakagawa, H., Katayama, S., Arata, Y., 1982. Weld metal cracking and improvement of $25 \% \mathrm{Cr}-20 \% \mathrm{Ni}$ (AISI) fully austenitic stainless steel. Trans. IWS 13, 41-58.

Montagnon, J., Moraux, J.Y., Hocquette, A., 2004. Application of thermo-calc to the developments of new heat-resistant alloy casting for specifics SPF-DB tooling. In: Proceedings of the 3rd European Conference on Super Plastic Forming, Albi, France, pp. 117-124 (Cépadues-Editions).

Ray, A.K., Sinha, S.K., Tiwari, Y.N., Swaminathan, J., Das, G., Chaudhuri, S., 2003. Analysis of failed reformer tubes. Eng. Fail. Anal. 10, 351-362.

Shankar, V., Gill, T.P.S., Terrance, A.L.E., Mannan, S.L.M., Sundaresan, S., 2000. Relation between microstructure, composition and hot cracking in Ti-stabilized austenitic stainless steel weldments. Metall. Mater. Trans. A 31, 3109-3122.

Shankar, V., Gill, T.P.S., Terrance, A.L.E., Mannan, S.L.M., Sundaresan, S., 2003. Solidification cracking in austenitic stainless steel welds. Sadhana 28, 359-382.

Suutala, N., Takalo, T., Moisio, T., 1979. The relationship between solidification and microstructure in austenitic and austenitic-ferritic stainless steel welds. Metall. Trans. A 10, 512-514.

Wu, X.Q., Jing, H.M., Zheng, Y.G., Yao, Z.M., Ke, W., Hu, Z.Q., 2000. The eutectic carbides and creep ruptures strength of $25 \mathrm{Cr} 20 \mathrm{Ni}$ heat-resistant steel tubes centrifugally cast with different solidification conditions. Mater. Sci. Eng. A 293, 252-260. 